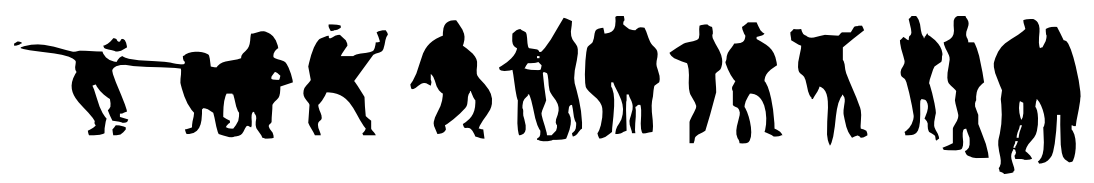

\section{Le TNI à I'école primaire : entre contraintes et engagement}

François VILLEMONTEIX(EMA, Cergy-Pontoise), Jacques BÉZIAT(FRED, Limoges)

RÉSUMÉ • Cet article marque une étape d'une recherche sur les usages et non-usages du tableau numérique interactif (TNI) par l'enseignant à l'école primaire et sur ses modes de scolarisation. A partir d'entretiens menés avec des enseignants déclarant une pratique routinière, nous inventorions et analysons les adaptations et les ajustements qu'ils réalisent afin de s'approprier ce dispositif technique, à la lumière d'un modèle d'analyse systémique en construction. Ces enseignants font face à des aléas et à un ensemble de contraintes qu'ils adaptent ou auxquels ils s'adaptent.

MOTS-CLÉS • TNI, tableau numérique, TICE, informatique, école primaire, pratiques d'enseignement, contraintes.

ABSTRACT • This articleis a step ina research on the use andnon-use of interactive whiteboard(IWB) in primary schooland its inclusion in the primary school.Frominterviews withteachersreporting aroutine practice, we recordand analyze adaptations and adjustments they maketouse this technical device. We build on a system analysis model being created. These teachers face troubles and a set of constraints to which they adjust or adapt.

- KEYWORDS - IWB, interactive whiteboard, computer science, primary school, teaching practices, constraints. 


\section{Introduction}

Parmi les dispositifs numériques disponibles pour la classe, le TNI a un statut particulier : proche du tableau classique par sa forme et la position qu'il occupe dans la classe, il permet aux enseignants de mobiliser rapidement un savoir-faire déjà en place pour son accueil et sa mise en œuvre. Plus que tout autre dispositif numérique, les enseignants possèdent un cadre d'accueil en classe du TNI, à la fois pratique et idéologique.

Sur ce point, se joue peut-être un paradoxe : jusqu'à quel point ces schèmes d'usages déjà rodés retiennent-ils l'innovation potentielle permise par le TNI? Autrement dit, doit-on penser que les plans d'équipement en TNI, dont l'objectif annoncé - du moins dans certains discours marketing - est de porter l'innovation pédagogique par le numérique au sein de la classe, sont susceptibles de renforcer des formes magistrales et classiques d'enseignement?

Les entretiens que nous menons depuis 2012 avec des enseignants bénéficiant de cet équipement suggèrent une réalité plus nuancée : au-delà des variations instrumentales liées au remplacement du tableau noir ou blanc par un TNI, l'évolution de leurspratiques professionnelles d'enseignement dépend de représentations qu'ils se font de l'éducation scolaire et joue surleurs modes d'appropriation du TNI. Les analyses des entretiens laissent apparaître des tendances dans les habitudes de travail, la préparation de leurs ressources, la gestion de leur environnement de travail. Les enseignants n'en sont plus à une phase de découverte, ils ont adopté le TNI, adapté leur activité d'enseignement à cet outil technique et reconfiguré ce dernier à celle-ci. Ils ont développé leur environnement de ressources, qu'ils organisent selon leurs propres normes. Ils les enrichissent progressivement et se faisant, maintiennent leur l'engagement dans l'utilisation de l'instrument. Ainsi donc, l'engagement individuel dans une forme d'instrumentation avec une technologie informatisée procède d'un choix coûteux et lourd, mais contient ses propres ressorts.

A partir d'un état de questions relatives au TNI et à la place qu'il occupe dans les stratégies institutionnelles d'équipement des écoles au niveau central puis local depuis plus d'une dizaine d'années, ce texte présente un éclairage sur une approche des processus à l'œuvre lorsque cette technologie est introduite dans l'univers professionnel des enseignants, contribuant à la construction d'une problématique centrée sur l'enseignant dans un système complexe. Il précise la méthodologie utili- 
sée, délivre les premiers résultats des analyses conduites, les discute et propose enfin quelques perspectives de recherche.

\section{A propos du TNI}

Le TNI constitue un dispositif complexe proposant de nombreuses potentialités mais qui ne l'écartent pas radicalement de sa filiation avec le tableau noir et le paradigme de la pédagogie transmissive. Cependant au cours de ces dix dernières années, il demeure un équipementsur lequel les collectivités ou les gouvernements ont largement investi.

\subsection{Ce qu'il est possible de faire avec le TNI}

Le TNI présente une diversité d'outils d'écriture, de capture d'images, d'animation de schémas et masquage par un rideau, de zoom, de manipulation d'objets, de reconnaissance de caractères, etc. Sur un même support, il est possible de regrouper plusieurs médias et de leur donner vie, avec l'adjonction éventuelle de comportements. L'ensemble des traces laissées et des documents produits peuvent être mémorisés, pour des enrichissements ou diffusion éventuels(Boulc'h et Baron, 2011). Un progiciel accompagne ce dispositif et représente le cœur du TNI. Il est en général piloté par un ordinateur ou directement par un vidéoprojecteur dans les modèles les plus récents. Il offre tout d'abord les fonctionnalités d'un logiciel classique de PréAO, libérant l'enseignant de l'espace contraint du tableau noir par la multiplication à l'infini de calques de présentation. Les possibilités de présentation de contenus, multimédias et interactifs sont nombreuses, mais leur mise en scène en direct selon un scénario mérite d'être anticipée du fait de nombreuses contraintes techniques (Villemonteix et Stolvijk, 2011).

Le TNI constitue un environnement auquel, par ses affordances, l'utilisateur s'adapte (Gibson, 1979). L'enseignant, selon sa perception des propriétés du TNI, constitue un espace de possibles, dans lequel il peut naviguer et qu'il prend en compte selon les fins qu'il se fixe (Morineau, 2001).

\subsection{Du tableau noir au TNI, continuité du paradigme transmissif}

Le tableau de classe caractérise l'espace scolaire. Il constitue depuis fort longtemps le complément ou le prolongement de la parole du maître jouant également un rôle de support au tâtonnement épistémique (Billouet, 2007). L'arrivée du tableau numérique ne réinterroge pas spécifi- 
quement la position traditionnelle de l'enseignant et n'amène aucun bouleversement immédiat dans la façon d'enseigner (Somekh et al., 2007). Le tableau reste un organisateur spatial et le geste pédagogique, basé sur l'alternance entre transmission simultanée, exercices et remédiation individuelle, n'est pas modifié. L'instrument sert en quelque sorte « l'ambition transmissive de l'école ».

D’une manière générale, les enseignants et les élèves apprécient beaucoup la présence et l'utilisation des TNI en classe. De nombreuses études ont été menées en Grande-Bretagne sur le TNI (BECTA, 2005), pour autant, il est difficile d'affirmer l'impact significativement positif du TNI sur les performances des élèves (Jeunier et al., 2005). Une étude approfondie sur les deux degrés, croisant des données d'entretiens, d'observations et de questionnaires sur un nombre important d'établissement ne parvient pas à attester de preuves précises quant à la pérennité des apprentissages (Higgins et al., 2005). Une autre étude montre que les modalités d'utilisation de l'outil ont une incidence sur les dynamiques d'apprentissage (Duroisin et al., 2011) sans pouvoir attester d'un lien significatif entre la progression des performances des élèves et l'utilisation réitérée du TNI.

Le programme britannique de développement des TIC a donné lieuà un programme de formation mettant l'accent sur une utilisation dans une pédagogie active. Mais les analyses de pratiques ont montré qu'une pédagogie transmissive ou expositive (documents, démonstrations), favorisant le texte (quizz) et la remédiation (exerciseur)s'est plutôt développée, au détriment d'une pédagogie active basée sur la production, l'expérience et la confrontation d'idées (Betrancourt, 2007).

Dans certains cas, quelques résultats attestent de l'intérêt d'une projection et d'une manipulation directe. C'est le cas des objets mathématiques et géométriques interactifs, dont les manipulations en classe jouent essentiellement sur le comportement, la participation et l'attention des élèves (Averis et al., 2005). Ceci se vérifie lorsque les élèves manipulent directement les objets qui leur sont présentés (Thompson et Flecknoe, 2003). L'exposition à des objets visuels ou sonores manipulables sur un plan vertical donne également lieu à une meilleure conscience phonologique dans l'apprentissage de l'anglais en primaire (Magnat, 2012).

\subsection{Le TNI dans les stratégies institutionnelles d'équipement}


EnFrance, lapromotiondecetoutil a probablement été influencée par la politiqued'équipementmassifdes écolesbritanniques (Chaptal, 2010). Plusieurs initiatives locales ou nationales ont donné lieu à l'attribution de TNI au cours des 10 dernières années dans les classes primaires. Citons le cas de l'opération « 1000 visio »(MEN, 2008), ou le plan «Ecole Numérique Rurale » (Plan ENR) où l'état et les collectivités locales ont cofinancé des configurations complètes. Ce plan a été considéré comme une réussite du point de vue de la cohérence de l'offreet de la cohésion des acteurs, mais comme un échec en termes d'accompagnement de l'offre ressources numériques, jugé insuffisant (IGEN, 2011).

Selon l'enquête annuelle ETIC, diligentée par le ministère de l'éducation nationale, en 2010, 23\% des écoles élémentaires disposent d'un TNI (MEN, 2010), ce qui laisse la France loin notamment derrière la Grande-Bretagne où toutes les classes sont équipées, le marchéétant arrivéà saturation. Contrairement à la France, le gouvernement britannique n'a pas attendu que la recherche atteste de la valeur ajoutée du TNI pour s'engager, dès 2004, dans une politique d'équipement généralisée (Arnott, 2004).Il revient sur celle-ci actuellement, compte tenu, d'une part des décisions politiques de l'administration Cameron à l'égard du BECTA, d'autre part d'un récent rapport de la Royal Society (Royal Society, 2013), qui pointe le manque de résultats probants d'une politique de diffusion massive de technologies et d'une vision très orientée « usages ».

La question de la formation à ces instruments reste cependant centrale et notamment en formation initiale. Plusieurs recherches convergent sur les représentations que des étudiants en éducation se forgent à propos du TNI et des technologies. Entre naïveté ou enchantement (Béziat, 2011) et méfiance, penser de cette manière est risqué selon Baron et Boul'ch, dans le sens où, confrontés réellement à l'outil sur le terrain, ces futurs enseignants risquent d'éprouver une grande déception. Le risque serait alors qu'ils se détournent des technologies en général (Baron et Boul'ch, 2012).

\section{Environnements et contraintes pour l'école primaire}

L'école primaire propose un cadre d'adaptation scolaire des TICE spécifique et contraint (Béziat et Villemonteix, 2012). Pour le TNI en particulier, et ce, malgré sa congruence avec le tableau classique, son utilisation à ce niveau s'avère complexe ce qui n'a pas empêché les collectivités locales françaises d'avoir fait des acquisitions parfois massives de ce dispositif technique, depuis près d'une dizaine d'années. Les usages restent parfois en deçà des attentes du fait de l'existence de facteurs de contexte interdé- 
pendants, déjà repérés par la recherche (Dwyer et al., 1994) ; (Baron et Bruillard, 1996); (Cuban, 2001); (Daguet et Wallet, 2012). Nous posons dans cette section un modèle de contraintes, par effet de synthèse sur ces travaux et de manière heuristique. Il part du principe que la pratique de classe est le produit d'un processus complexe et multifactoriel qu'il convient d'appréhender de manière systémique. Les pratiques de classe, et les discours sur ces pratiques, sont des points d'observation permettant de mettre en perspective les facteurs contrariants ou favorables à des usages des technologies informatisées en classe.

\subsection{Interventions institutionnelles dans le domaine des TIC}

Du point de vue administratif, l'école primaire française n'est pas un établissement et ne dispose d'aucune autonomie financière ni juridique, les collectivités locales ayant compétence dans les domaines de l'équipement et des infrastructures et l'état en termes de pilotage pédagogique. Les collectivités ont obligation de répondre aux prescriptions de l'éducation nationale (le B2I, par exemple) mais déterminent localement la hauteur des investissements selon les marges de manœuvre disponibles, variables selon les territoires. Ceci donne lieu à d'importants déséquilibres en termes d'équipement informatique et la couverture nationale ${ }^{1}$ reste encore en deçà de celle des voisins européens².

Ajoutons que, dans le meilleur des cas, les communes équipent, en appui des recommandations fournies par l'éducation nationale, en général des inspecteurs locaux accompagnés d'un enseignant expert l'animateur TICE (ATICE) -, mais il n'est pas rare que les communes équipent unilatéralement, sans qu'un processus de décision collective ait pu avoir lieu, certains enseignants se voyant attribués des dispositifs techniques non demandés.

La sociologie de l'innovation a montré que s'agissant de l'introduction d'une innovation dans une organisation, un dispositif de concertation réunissant acteurs et actants autour d'un traducteur pouvait s'avérer nécessaire pour traiter les controverses et mettre en place des terrains d'entente (Akrich et al., 1988). C'est dans cette perspective que le projet OPPIDUM, pour Observatoire des Pratiques Pédagogiques Innovantes et des Usages du Multimédia, a été mis en œuvre dans la ville de Saint-Maur-des-Fossés (Baron et al., 2011). L'expérience s'est montrée intéressante dans la tentative de mise en place d'un modèle de pilotage concerté entre une collectivité et l'état, un laboratoire universitaire jouant le rôle de traducteur ${ }^{3}$. 
Cependant, des pratiques très divergentes parfois même au sein d'un même département (Villemonteix, 2011) sont caractéristiques de contextes financiers variés, mais aussi de stratégies locales sur le pilotage de l'activitééducative. Certaines communes ajoutent des offres locales d'accompagnement et de formation via des ressources numériques associées aux matériels achetés sous la forme de forfaits, en direction des familles. Si ce scénario devait progresser, se poserait alors la question du contrôle par l'État de l'équité territoriale de l'offre éducative.

Une autre considération de contexte est à prendre en compte, c'est celui de la légitimité des pratiques instrumentées à l'école qui ne s'adosse à aucun élément de programme de l'école primaire. Or les programmes sont à la base du pilotage de l'activité enseignante et l'existence d'un référentiel de compétences annexé aux programmes, le Brevet Informatique et Internet (B2i) ne semble pas suffire pour faire levier. Ce référentiel centré sur l'appropriation des compétences réduites à des savoir-faire et des procédures, amène à une impasse sur les savoirs en jeu. Les enseignants ont à construire un ensemble structuré et cohérent de représentations constituant une forme de conscience de ce dispositif (Fluckiger et Bart, 2012).

\subsection{Les processus de diffusion des TICE à l'école primaire, approches systémiques}

Rendre la pratique instrumentée avec les technologies intelligible, amène à prendre en compte la grande variabilité des contextes locaux de manière systémique. "En matière de recherche, l'approche systémique a mis l'accent sur la nécessité de prendre en compte la globalité des variables qui peuvent agir sur le processus éducatif plutôt que de se contenter de manipuler un nombre limité de variables isolées de leur contexte. »(Depover, 2009).

Dans la littérature, différentes approches permettent ainsi de guider les études sur les discours des acteurs impliqués dans l'appropriation d'une innovation technologique et l'évolution de leurs pratiques professionnelles. Le modèle «Pédagogie, Acteurs, Dispositif, Institution » (PADI) développé par Wallet est notamment mobilisé pour étudier les non-usages (Daguet et Wallet, 2012). Il permet de focaliser davantage sur l'état d'un système que sur un processus dans lequel s'inscrit l'activité pédagogique. Les processus de généralisation des technologies en milieu éducatif reposent sur des leviers hétérogènes et interdépendants difficiles à appréhender dans leur ensemble, compte tenu de la variété des représentations, des acteurs et des structures. 
D'autres modèles permettent d'appréhender les phases des processus d'appropriation d'instruments informatisés en se situant au niveau de l'enseignant dans son contexte. Le modèle ACOT, issu de l'étude de Dwyer et ses collègues (Dwyer et al., 1994) sur le dispositif Apple Classrooms of Tomorrow (ACOT) aux USA et au Canada, pointe plusieurs étapes à franchir dont chacune prend du temps et nécessite des conditions pour conduire à la suivante : entrée, adoption, adaptation, appropriation, invention. Selon les chercheurs, on en reste souvent aux toutes premières phases du processus faute de soutien suffisant, ou il y a abandon, si l'usage des dispositifs est jugé trop coûteux en temps et en effort ou trop en opposition par rapport aux pratiques traditionnelles.

Depover et Strebelle (Depover et Strebelle, 1997) prennent également cet angle de vue et considèrent trois phases dans les processus d'innovation pédagogique : l'adoption, l'implantation et la routinisation. Les auteurs proposent un modèle d'analyse complexe du processus s'articulant autour de deux axes complémentaires, un axe dynamique, celui des « intrants », des processus et des «extrants » et un axe topologique, référé aux différents sous-systèmes par rapport auxquels le processus d'innovation s'inscrit, du plus spécifique au plus englobant : la classe, l'environnement scolaire, le circuit administratif et prescriptif et les variables présentes dans l'environnement immédiat du système éducatif (De Lièvre et Moulin, 2008).

Ainsi, dans le modèle AFRI présenté ci-dessous, nous prenons en compte deux niveaux de variables liés à l'adaptation scolaire des technologies informatisées: celui des enjeux et celui des composantes de l'activité en classe.

\subsection{Modèle d'analyse AFRI (axes, formation, ressources, implications)}

Le modèle que nous présentons ici vise à caractériser les relationsentre l'activité enseignante mobilisant une innovation technique et son environnement. Dans le contexte scolaire, l'activité consistant à conduire une classe avec des technologies est reliée aux composantes de cet environnement (idéologique, institutionnel, social et technique). Elles se manifestent par des outils (outils, matériels), des individus (sujet, soi ; la communauté-les autres), des normes (règles-programmes), susceptibles d'évolution. De son côté, l'enseignant, qui agit dans un collectif et une structure (l'école), fait face à des contradictions et des contraintes pour agir et donner sens à son activité. Dans une perspective d'instrumentation 
durable de son activité (Rabardel, 1995), il répond à plusieurs enjeux qu'il identifie ou qu'il se fixe. Le modèle AFRI distingue quatre types d'enjeux et les réponses qu'il fournit à ces enjeux conditionnent l'accueil et les utilisations de ces innovations technologiques : un enjeu axiologique et de formationun enjeu de ressources et un enjeu d'implication.

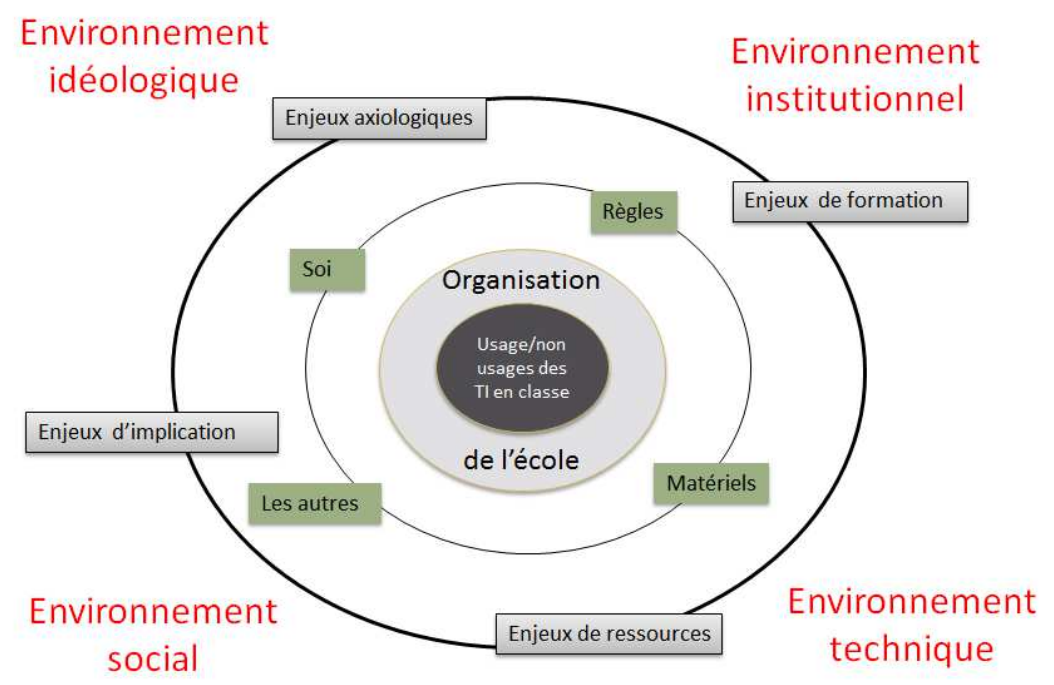

Figure 1: Système de contraintes pour l'intégration des TICE en classe, modèle AFRI

Sur le modèle, deux groupes environnementaux sont identifiables : celui sur lequel les enseignants ne peuvent pas directement agir (environnements institutionnels et techniques), et celui sur lequel les enseignants peuvent directement agir (environnements idéologique et social).Chacun des facteurs (environnements, enjeux et contraintes) est nécessaire mais non suffisant pour permettre l'usage des technologies informatisées par l'enseignant en classe.

\section{Méthodologie}

\subsection{Prise d'appui sur le discours des enseignants}

L'objectif est d'identifier ce que l'enseignant repère comme contraintes des différents environnements ciblés et d'identifier les solutions qu'il adopte pourles écarter ou s'en arranger. L'enseignant entreprend des actions, fait des choix, mais agit dans un milieu, un réseau de relations plus large, qui agit sur lui et sur lequel il agit. 
Afin de les identifier, nous avons privilégié une méthode de collecte de données qualitatives, en réalisant des entretiens semi-directifs d'une durée approximative d'une heure. Ces entretiens ont été enregistrés et soumis à une analyse de contenu sous MODALISA. Certains des enseignants ont complété ces entretiens par des démonstrations sur leur TNI de classe. A partir de ces discours, nous avons procédé par tas thématiques au fil de l'eau (Bardin, 1977), que nous avons ensuite recentrés sur des thèmes génériques, en lien avec le modèle AFRI.

Ainsi, nous avons privilégié une méthode qualitative, à visée compréhensive et descriptive qui s'inspire de l'approche inductive de Miles et Huberman (Miles et Huberman, 2003). Les pratiques déclarées des enseignants s'inscrivent dans le cadre quotidien et routinier de la classe. La mise à jour d'un système explicatif par le sens que donne le sujet à son action s'avère féconde, même si le sens ou l'intention déclarée par l'individu, ne constituent qu'un des éléments du système de causalité. Cet élément s'intègre dans le jeu des contraintes de l'action collective dans lequel l'acteur est inséré (Alami et al.,2009).

La grille d'entretien utilisée aborde plusieurs points : présentation de l'enseignant (ancienneté, pratiques personnelles des technologies); conditions de mise en œuvre du TNI (problèmes posés, solutions esquissées, acteurs mobilisés) ; rapportsà la formation (institutionnels, par pairs, autoformation); préparation des ressources (outils mobilisés, types de ressources, objectifs, méthodes); pratique du TNI en classe (activités développées, modalités de travail, activité des élèves) ; représentations du rôle des technologies enéducation.

Six entretiens sont traités dans cette étude, encore exploratoire et à valeur heuristique. Nous mobilisonsle modèle AFRI comme analyseur des témoignages présentés dans ce texte. A terme, ce travail de recherche nous amènera à dégager différents profils enseignants d'accueil du TNI en classe, et à en discuter les spécificités, ainsi qu'à distinguer les pratiques évoquées des représentations ou croyances sur ces pratiques.

\subsection{Présentation des enseignants}

Les entretiens concernent deux hommes et quatre femmes, situés dans deux départements, sur des territoires différents sur le plan sociologique.

- Le premier enseignant (e1) travaille dans une classeélémentaire d'une école d'une zone défavorisée de la banlieue sud de Paris, classée en zone d'éducation prioritaire (ZEP). Cette école répondant à des critères 
sociaux spécifiques, l'effectif d'élèves de sa classe se limite à 22. Il travaille depuis 3 ans avec un TNI. L'enseignant se déclare féru d'informatique. Il dispose d'un blog, qu'il co-conçoit avec ses élèves. Il présente cet outil comme l'ENT de la classe accompagnant les élèves dans et hors l'école. Il n'a plus de tableau noir. Il utilise massivement le TNI, mobilise de nombreuses ressources d'internet. Il utilise les calques, les fonctions de cache, d'annotation, de capture d'image en permanence. Le plus souvent Il garde certaines traces qu'il poste sur le blog. Il fait utiliser le TNI aux élèves. Il n'utilise pas de manuel numérique mais compose et anime en utilisant le logiciel de TNI et d'autres logiciels en parallèle (lecteur vidéo, son). Il utilise la bibliothèque pour y placer ses propres ressources.

- La deuxième enseignante (e2) travaille dans une école d'une commune aisée du même département.Elle enseigne depuis 29 ans et est en poste depuis 7 ans dans l'école. Elle utilise le TNI depuis 4 ans. Elle déclare des compétences fragiles en informatique, mais utilise régulièrement la salle informatique de l'école. Elle utilise le TNI pour projeter, pour annoter des documents et en garder trace. Elle a disposé le TNI en fond de classe et le fait utiliser très régulièrement aux élèves qui ont pris en charge son fonctionnement. Elle n'utilise pas la bibliothèque du logiciel TNI.

- La troisième enseignante (e3) travaille dans une école située dans une autre zone d'éducation prioritaire. Elle a presque toujours enseigné dans cette école, puisque sur 20 ans d'ancienneté, elle a passé 18 ans dans l'école.Elle utilise également des baladeurs MP3 (un par élève) pour l'apprentissage des langues vivantes. Le vidéoprojecteur est installé au plafond. Elle utilise le logiciel du TNI et, en parallèle, le logiciel de traitement de texte, pour préparer ses séances. Elle utilise des manuels numériques mais en importe des captures dans le logiciel TNI. Elle n'utilise pas la bibliothèque de ressources mais a organisé ses ressources par disciplines et par thèmes.

- La quatrième enseignante (e4) enseigne depuis 15 ans dans une école située dans un quartier résidentiel d'une commune populaire. Elle est maître-formatrice et participe ainsi à la formation des nouveaux enseignants. Elle utilise le TNI depuis 3 ans. Ses compétences et connaissances lui permettent de mettre en œuvre le TNI avec aisance. Elle crée ses propres manuels numériques qu'elle utilise en permanence. Elle utilise essentiellement le logiciel fourni avec le TNI et met en œuvre l'ensemble de ses fonctionnalités, les outils d'annotation, les calques, la capture, les caches et utilise la bibliothèque du logiciel pour placer ses ressources. Elle utilise un blog de classe qu'elle nomme le « cartable numérique » qu'elle a conçu avec l'aide de l'ATICE. 
- Le cinquième enseignant (e5) enseigne depuis 10 ans et travaille depuis 9 ans dans une école située dans une ville populaire de l'est parisien. Il dispose de postes informatiques dans sa classe (6) utilisés fréquemment par les élèves. Il détient un TNI depuis 5 ans, qu'il a obtenu par projet. Il l'a utilisé en cycle 3 pendant 3 ans et depuis deux ans l'utilise en CP. Il a numérisé tous les manuels élèves et recompose, en fil de l'eau, des ressources d'appui pour l'exposé de leçons et la réalisation d'exercices.

- La sixième enseignante (e6) travaille dans le centre-ville d'une grande ville de province. Elle est enseignante depuis vingt ans. Cette année, elle a une classe à double niveau de 31 élèves: CE2/CM1. A titre personnel, elle se considère comme une très faible utilisatrice des TIC, elle est très attachée aux moyens classiques de communication, d'achat, de relation. Dans la classe, elle a commencéà utiliser le TNI au moment de la dotation de sa classe, depuis deux ans, et depuis, n'utilise que ce type de dispositif numérique. Durant la dizaine d'année qui a précédé l'installation du TNI, elle a eu quelques tentatives d'usages des technologies informatisées, sur un mode d'opportunité, en fonction du matériel présent. Son levier principal d'usage des technologies informatisées en classe s'appuie sur la prise de conscience des enjeux d'une formation au numérique pour les élèves d'aujourd'hui.

\section{Résultats}

Les entretiens font apparaitre différents niveaux de tensions et de contraintes qui structurent, orientent, limitent, encouragent certains usages des technologies informatisées en classe. Notre approche heuristique, par le système de contraintes (cf. figure 1), permet d'organiser les témoignages retenus ici. Les retours d'entretiens sont organisés dans les sections suivantes selon le modèle AFRI : d'abord les composantes de l'activité du modèle, ensuite les enjeux liés à l'adaptation scolaire des technologies informatisées.

\subsection{Les composantes de l'activité}

\subsubsection{Soi}

De manière générale, le TNI, objet numérique incontournable puisqu'en place du tableau classique, fait évoluer les enseignants sur leurs propres représentations des technologies informatisées en classe. Les questions de dynamique personnelle sont liées à l'implication dont sont capables les enseignants pour accueillir un nouveau dispositif technique. Ici, les enseignants insistent sur le volontariat nécessaire, et aussi sur certaines 
formes d'adoption définitive du dispositif et d'acceptation des évolutions instrumentales et pédagogiques imprimées par le TNI.

Une volonté affirmée apparaît dans les témoignages recueillis, de faire en sorte que le TNI soit fonctionnel et soit utilisé, malgré les contraintes posées et les solutions adoptées, contredisant parfois les normes de sécurité : «(e2) Parfois ceux qui sont derrière ne voient pas facilement. Ils se déplacent, ils savent qu'ils peuvent le faire»; "(e4) Quand quelque chose qui fonctionne pas, au niveau de sécurité avec des fils qui traînent par terre, la solution, c'est «tu t'en sers plus» et ça ce n'est pas possible. » (e5) «En fait il y a des regroupements, on est toujours en mouvement. Je suis obligé de brancher débrancher. Je n'ai pas de bluetooth malheureusement ».

Le TNI amène aussi à certaines remises en cause, et conduit à d'autres évolutions, d'autres manière de voir son rapport à l'informatique en classe et, d'une certaine manière, son propre rapport à la classe : (e1) «C'est bien, ça nous ouvre à tout. Plus loin, faut qu'on voit nous nos propres limites. Il faut qu'on se modernise il faut aussi qu'on revoit notre enseignement. Les enfants sont plus attirés par ces objets qu'on ne connaît pas. L'informatique en général j'ai pris le truc tout de suite.Les enfants savent plein de choses, ils nous montrent»;(e4) "Il y a 3 ans, je voulais passer l'entretien de direction et l'arrivée du TNI m'a fait changer d'avis et commencer autre chose en classe. (...) Le TNI me tient ici $»$.

Parmi les témoignages retenus ici, une enseignante plus en retrait sur les technologies informatiques reconnait une plus-value au TNI. Ses pratiques sont en tension entre le maintien d'un fonctionnement classique et l'une exploitation des potentialités du TNI :(e6)«là, je manque complètement de compétences. (...) oui, oui,pour le moment je fonctionne à l'ancienne et ça avance très bien quoi (...). (...) toutes les matières, histoire de l'art, n'en parlons pas. Moi je ne pourrai pas faire histoire de l'art si je n'avais pas ça».

\subsubsection{Les autres}

Ce que font apparaître les items de ce groupe thématique, c'est un certain isolement de l'enseignant face au dispositif technique, d'autant plus grand qu'il est lui-même fragilisé par la présence de ce dispositif. En creux, les propos font aussi apparaître le besoin d'un accompagnement spécifique aux effets liés à l'arrivée de technologies informatisées dans l'école, allant contre l'idée qu'il est normal que le TNI soit utilisé avec facilité par tout le monde. 
Les soutiens requis dépendent assez nettement des compétences déclarées et repérées des enseignants interviewés. Les enseignants évoquent essentiellement cette question en termes de déficit. Il tient à des considérations techniques, ouportant sur le terrain de la reconnaissance.Sur le plan pédagogique, aucun enseignant ne déclare requérir de soutien spécifique.

Sur le plan technique et logistique, les enseignants expérimentés et disposant de compétences informatiques robustes prennent des initiatives pour que leur système fonctionne et que leur activité se maintienne. Pour certain, le soutien bienveillant du directeur ou de la directrice est souligné : (e1) «Je vais directement voir les gens. La directrice m'a toujours soutenu. Ça la dépasse, donc si vous avez des problèmes, vous réglez ce sera plus simple».

Ce soutien aux initiatives peut être aussi limité : (e2) «Dès fois j'emporte l'ordinateur, je me dis que si je me le fais voler... C'est ma directrice qui me l'interdit» ou inexistant, faute d'intérêt: (e3) "Pas d'aide du tout. Elle n'intervient pas dans notre travail. On travaille ensemble. Elle ne sait pas de quoi on parle, elle ne peut rien suggérer ».

Quoiqu'il en soit, le simple fait qu'ils prennent en main la question informatique (le TNI) les place au rang de personne-ressource pour les collègues: (e4) «Plein de gens pensent que je suis une ressource informatique. Je montre à mes collègues ».

Sur le plan de la reconnaissance institutionnelle, un certain sentiment d'isolement se fait sentir chez certains à l'issue des opérations de dotation, interrogeant finalement les rationalités des acteurs à participer aux opérations de ce type, à leur démarrage: qu'en attendent-ils ? Quel est le contre-don? (e2) «J'ai pas de suivi, ni surveillance. Personne n'est venu voir pour savoir comment ça fonctionnait. (...) Il y a eu un petit suivi dans le cadre de la visioconférence la première année, je suis alléà Educatice. On ne nous demandait pas des comptes "est-ce que ça vous plaît, est-ce que vous vous sentez à l'aise ", mais c'est tout"; (e5) "On nous confie de matériel qui vaut plusieurs milliers d'euros, mais on nous demande plus rien après».

Chez d'autres le recours à des soutiens extérieurs n'est pas d'actualité. Là encore le niveau de maîtrise des instruments peut justifier cette nondemande : (e1) «Non, on s'est croisé (avec l'ATICE), mais j'étais déjà bien en route, alors non. Il est revenu faire une animation pédagogique dans la classe 
cette année, je n'avais pas besoin d'être présent. Je ne lui ai pas demandé de ressource. Je ne sais pas où sont les torts ».

\subsubsection{Matériels}

Les questions matérielles sont, à l'évidence, fondamentales dans un contexte d'instrumentation des pratiques scolaires. Ces enjeux matériels sont le lieu de discussion entre les collectivités et les écoles. Le plus souvent, le TNI est installéà la place de l'ancien tableau, ou à l'endroit le moins gênant, selon les conditions de lumière par exemple. Faire poser le TNI à un autre endroit que celui prévu pour le tableau peut s'avérer difficile. Autrement dit, ce sont des considérations matérielles (la lumière, la place) ou coutumières (la place habituelle du tableau) qui déterminent la façon dont le tableau sera installé, à la classe de s'adapter. Autrement dit, cela revient le plus souvent à renforcer l'organisation traditionnelle des classes.

La question des moyens est omniprésente dans les témoignages relevés. Soit par l'expression d'un manque, de questions matérielles d'installation, de risques, d'état de fonctionnement, de rapidité de réponse du dispositif, de déficit de maîtrise technique du dispositif, d'adaptation de la vie de la classe à ce dispositif... Dans tous les cas, ces points impactent sur la pratique quotidienne de la classe : (e1) «Le Trackpad de l'ordinateur n'a plus fonctionné, j'ai dû acheter moi-même une souris, sauf que j'ai pas de crédits non plus. Tous les trucs que j'achète, c'est moi qui les achète. Ma directrice me rembourse parfois. (...) J'ai fait venir des gens pour constater l'état de dangerosité de l'installation, risque de chute, de casse matérielle, des prises qui se branchent un peu partout (...)». ; (e4)«Il y a un enfant délégué de tableau, qui prépare, lance l'ordi, toute les semaines ça change. Il doit faire attention à tous les fils».

Ces problèmes d'installation peuvent devenir un problème pour le fonctionnement de la classe : (e1) «Il est au fond de la classe, du coupje le décale là (vers la fenêtre), mais ça leur enlève trop de jour si jamais je le laisse installé constamment. C'est cette table-là qui sert à l'installation... du coup ça prend du temps. (...) La position c'est au milieu là(vers la fenêtre) mais tout le groupe qui est là ne voit rien, à cause de la luminosité, donc ils avancent (...).Chaque matin, quand je prévoyais une séance sur ma clé et que je l'amenais ici, il fallait que je vérifie que tout fonctionne. "; (e2) «On est dans la largeur, parce que les focales ne sont pas suffisantes. Après ça pose un problème de déplacement. Je travaille beaucoup ça en début d'année. Je ne peux plus passer aussi facilement derrière les uns ou les autres. (...) Il y avait au 
début un sens de circulation, parce qu'il y avait des câbles dans tous les sens. A une époque c'était même dangereux, puisqu'il y avait un câble à la hauteur de la gorge des enfants devant la porte de secours. J'ai du scotch de théâtre pour fixer les câbles au sol».

La question des moyens est largement contrainte par la volonté ou la capacité de financement de la commune : (e3) «TNI dans l'école depuis 2 ans. Il est payé par l'EN et installé par la mairie. C'est l'inspectrice qui a demandé. Pas de difficulté de la part de la ville. Ils sont plus réticents pour en acheter d'autres. (...) J'ai fait une présentation devant la mairie pour voir, en situation, à l'initiative de l'I3EN pour inciter la mairie à financer.» Ce financement du matériel est déterminant sur l'accessibilité aux ressources numériques: (e3) «On s'aperçoit que quand on n'a pas un TNI dans sa classe, on ne peut s'y mettre, c'est difficile à prêter. (...) Comme il est là-haut, je ne le recalibre qu'au retour de vacances quand je réinstalle le vidéoprojecteur. Je n'ai pas d'ombre et je l'utilise quand je veux, 3 mn, 1 heure ou pas du tout. »; (e1) «Il y en a eu 4 ou 5 donnés par l'académie. En fait ils ne servaient à personne. Là-dessus, on nous a dit «soit vous nous faites la preuve que vous vous en servez, soit ils vont être donnés à une autre école».Je trouvais dommage qu'il s'en aille... (...). Mais il était dans la salle informatique, il n'était pas du tout dans ma classe. Ce que j'ai expliqué, c'est que personne d'autre de l'école ne s'en servait et que l'avoir dans ma classe était beaucoup plus simple que l'avoir dans la salle informatique[...]Depuis deux ans, la mairie m'a mis internet dans la classe. »

\subsubsection{Règles}

La présence du dispositif technique, essentiellement le TNI pour les enseignants enquêtés, fait évoluer la réflexion des enseignants sur les objectifs éducatifs poursuivis. Elle permet aussi de faire évoluer certaines intentions pédagogiques, certaines modalités de travail en classe. Ici, deux aspects émergent des témoignages: l'évolution de certaines pratiques d'enseignement et le sens de ces évolutions.

Les enseignants se lancent dans des actions, des productions, et doivent alimenter en contenus, en occasion d'usages : (e4) « J'ai voulu un blog pour l'école pour la classe transplantée. [...] C'est la seule technologie que je mettais en place. (...) Ce sont les enfants qui font des comptes rendus de journée, sur 15 jours, des textes libres [...]C'est en fonction des projets qui se présentent.» 
L'objectif est aussi de permettre aux élèves de développer leur esprit critique face aux technologies, aux informations qu'elles donnent, aux contenus qu'on y trouve : (e6) "Les idées couchées sur le papier, je trouve $q u$ 'elles viennent plus facilement que si on tape directement, voilà, moi la dessus je suis un peu resté vieille France.(...) et je pense que l'on doit apprendre ça aux enfants (ndlr: avoir de la méthode) et puis ça leur apprend à avoir l'esprit critique par rapport à ce qu'affiche la machine».

\subsection{Les enjeux}

\subsubsection{Enjeux de formation}

Le sentiment d'avoir été insuffisamment formé ou de l'avoir été de manière inadaptée revient fréquemment : quelqu'un vient montrer comment ça marche, puis l'enseignant doit découvrir seul comment s'en servir. L'autonomie par rapport aux technologies informatisées constitue sans doute le meilleur facteur d'adaptation.

Quatre enseignants relèvent certaines difficultés de maîtrise ou de manipulation des matériels et des interfaces. Ils reconnaissent des difficultés et leurs limites sur certains aspects manipulatoires: (e4) "Je ne sais pas bien faire pour les cartes de géo vierges »; (e3) "Quand je fais une séance avec le logiciel WS, le fait de transporter le fichier sur un disque extérieur me fait perdre tous les liens (internet, vers un autre document). J'ai la base du doc, mais plus les liens».

La formation technique est jugée nécessaire et le terme informatique est évoqué :(e3) «Si on n'a pas une maîtrise de l'informatique, on est vite dépassé, on ne peut pas voir l'utilité du TNI. Il faut une connaissance préalable. (...)»; (e4) «Les connaissances informatiques sont indispensables. Ça peut être tout simplement une erreur de connexion, de branchement, de fils, ou alors un problème de capacité de l'ordinateur, qu'il n'est pas assez puissant et que ça ne sert à rien d'insister. (...)»

Ce qui ressort, c'est un sentiment de bricolage et de débrouillardise et que l'appropriation se fait dans l'urgence, en contexte : (e6) «Enfin, moi je fais partie des gens qui ont un peu appris tout seul sur le tas à force d'essayer ».Les actions de formation sur site ciblant les besoins individuels sont privilégiés mais ne montrent leur efficience que dans une perspective d'accompagnement : (e6) « une personne ressource se déplaçait dans l'école et faisait une formation aux collègues de l'école, mais dans les classes[...] on s'est rendu compte d'une chose, c'est que des gens comme moi qui avaient déjà essayé de bricoler[...], on avait des demandes précises et on avait des réponses précises, 
et donc on pouvait avancer, les gens qui n'étaient toujours pas rentrés dans le système n'ont rien retiré de cette formation. »

Deuxenseignants (e1 et e5) n'éprouvent pas ce type difficultés. Peu intéressés par des formations délivrées par les personnels de l'institution, ils disent apprendre seuls il y a un intérêt ou que c'est nécessaire pour eux : (e1) «Dans ce domaine-là, je suis assez à l'aise pour aller chercher les réponses tout seul quand je rencontre un écueil ou une problématique». En revanche, les deux s'accordent avec les autres sur les formes non adaptées des formations aux contextes locaux et plaident davantage pour les échanges entre enseignants, contextualisés : (e1) «ce qu'il faudrait travailler, mais c'est plus en termes d'échanges que de formation, c'est la réalisation d'exercices, par l'échange: qu'est-ce que t'as fait comme exercice, ah oui, tu utilises cette fonction, c'est intéressant. »

Sur le plan du rapport à la formation des enseignants interrogés, quelques points apparaissent ici. L'acquisition de compétences instrumentales et d'habiletés constitue un préalable indispensable et paraît utile pour construire des représentations des pratiques possibles avec l'instrument. Cette demande s'enrichit par une autre, relevant de l'accompagnement fin, en situation, en réponse à des besoins locaux de perfectionnement des procédures. La prise de risques autonome vers la découverte de nouvelles fonctions ou de nouveaux instruments est le fait d'enseignants manifestant un degré de maîtrise plus important.

Dans l'étude du rapport à la formation, la nature des formations demandées serait à relier aux compétences et connaissances déjà acquises des enseignants. La demande semble s'affiner, elle porte sur des considérations didactiques et pédagogiques dès lors que certains schèmes d'utilisation sont présents. A ce niveau, c'est l'échange de pratiques qui semble être privilégié.

\subsubsection{Enjeux d'implication}

Les entretiens montrent que le TNI n'arrive pas sans bousculer certains repères dans la conduite de classe, certaines habitudes, certains modes de travail de l'enseignant. Il va amener les collègues d'une même équipe soit à demander de l'aide, soit à en proposer. Enfin chacun reconnait l'importance du temps consacréà produire ou à mettre à niveau leurs ressources de classe.

Sur les questions d'espace, le tableau ne se manipule pas comme un tableau classique: (e3) «Je suis en recherche pour ma position physique, déjà ; 
J'ai été obligée de moduler ma position physique. J'étais très rarement à mon bureau. Depuis 4 ans, j'y suis tout le temps. Comme il faut manipuler l'ordinateur et qu'on n'est pas en wifi, je suis obligé d'être à côté et d'être "branchée». Je ne peux manipuler l'ordinateur à un autre endroit. Ça change complètement ma façon d'être dans la classe et d'interagir avec les élèves. »

Des relations d'aide peuvent s'installer dans l'équipe. Certains enseignants usagers du TNI font des offres, parfois non relevées par leurs collègues: (e1) «Les premières années j'ai mutualisé, mais après personne ne s'en est servi. Chaque rentrée je leur ai proposé de laisser ma salle, leur brancher, mais jamais personne n'est jamais venu».Se joue ici le besoin qu'ont les enseignants qui se lancent, de créer des conditions collectives de réflexion pédagogique, de formation entre pairs.

Le TNI exige de l'enseignant un investissement réel, du moins au début, pour une montée des ressources sur supports numériques : (e6) « Je ne peux pas me permettre, j'veux dire, je peux pas passer trois heures à bricoler sur l'informatique pour une séance qui va durer une demi-heure, c'est pas possible, donc si techniquement, euh, je maitrise pas et je sens que ça va me prendre trop de temps.[...]oui, en fait, oui, il n'y a que 24 heures par jour, non c'est-à-dire que je me suis rendue compte d'une chose, c'est que... travailler avec les systèmes informatiques, enfin, moi, pour moi, à titre personnel, est chronophage, énormément chronophage[...]. J'ai transféré sur informatique tous mes corpus d'exercices ou de chose comme ça que je pouvais encore utiliser, que je stocke, et là, ben je peux reprendre modifier rapidement, etc... mais ça veut dire que pendant un certain temps l'été, j'ai passé un certain nombre d'heures à tout retaper, à tout mettre sur clé, alors que tout était papier avant." Le TNI conduitl'enseignant à revoir ses ressources, ses préparations de classe, à les porter au format numérique, et parfois, à devoir en repenser l'utilisation en classe, même pour les enseignants qui déclarent faire la classe comme avant.Ce tropisme vers l'ordinateur pose cependant problème en cas d'aléa technique: (e5)«Quand j'ai eu l'ordinateur qui n'a pas fonctionné pendant deux jours, c'était dur».

Le TNI peut ne pas être utilisé si, au minimum il n'y a pas accord de l'enseignant, un gré (Ingold, 2010) : (e6) «j'ai un collègue en haut, qui ne l'allume jamais, mais bon il a une classe de CP, c'est peut-être plus facile de s'en passer[...] ». Autrement dit, quelles que soient les compétences techniques initiales de l'enseignant et ses représentations personnelles sur les TICE, si l'enseignant décide de ne pas s'en servir, il ne s'en sert pas. Il n'y a pas d'appropriation sans un minimum d'implication de la part de 
l'enseignant. Dans les témoignages retenus ici, les enseignants usagers sont amenés à repenser la gestion spatiale de la classe, leur position devant les élèves. Le besoin d'échanger sur les pratiques les amène parfois à faire des offres de disponibilité auprès de leurs collègues, il ne suffit pas d'être convaincu, il faut aussi convaincre. Enfin, la gestion du temps personnel devient un facteur important dans l'appréciation des gains et des coûts liés à l'installation du TNI en classe.

\subsubsection{Enjeux de ressources}

Le remplacement du tableau classique par un TNI a des conséquences sur l'organisation, la gestion et l'utilisation des ressources pédagogiques de l'enseignant : le temps passéà les produire, à les porter au format numérique, sur leur accessibilité, sur la manière de s'en servir en classe, sur la manière de penser l'interaction avec les élèves. La centration sur ces ressources projetées et manipulées frontalement a également une incidence sur le renforcement de certains styles pédagogiques des enseignants.

Les enseignants établissent un rapport entre les contraintes des supports classiques et les facilités offertes par les supports numériques, plus confortables et rapidement accessibles : (e2) «Si on avait une mappemonde ou un globe terrestre, il faudrait refaire des explications à chaque fois. Les représentations sont visuellement impactantes. Je n'ai pas besoin d'aller chercher un $C D$ dans une armoire. Pour les élèves, il y a un côté magique».

L'activité de préparation du travail change de nature et c'est dans une perspective économique qu'elle est perçue :(e6) «sije veux partir sur un texte qui pose problème en grammaire, [...] plutôt que de copier au tableau, je prépare tranquillement chez moi et je l'affiche, l'intérêt c'est que c'est interactif, ... on peut rajouter, enlever facilement, sans avoir à effacer et puis surtout on peut garder en mémoire pour la séance suivante, ça pour moi, ça c'est vraiment un plus.»; (e1) «Beaucoup moins de manipulations de papier. En maths, c'est plus pratique, je n'ai plus besoin d'affiches. Moins de préparation, oui. En fait l'intérêt c'est d'avoir le même support qu'eux, sans avoir besoin de reproduire». La composition par bricolage existe mais prend une forme plus rationnelle, écartant parfois les instruments, la perspective est davantage la substitution que la complémentarité.

Par ailleurs la légitimation de l'utilisation du TNI se fait par le manuel. Le TNI permet de le magistraliser et les manipulations permises par l'instrument sont perçues comme des valeurs ajoutées pédagogiques et didactiques:(e3) «Il n'y a plus de raison [pour l'élève] d'être perdu. Le TNI 
permet d'agrandir la page et de manipuler. Ce qui est sur le cahier est sur le TNI. La transparence permet de bien repérer, mieux qu'avec une vraie équerre. Les compétences s'améliorent avec le TNI. Les élèves ont besoin de repères, que ce soit à l'identique».

Deux entretiens (e4 et e5)mettent en évidence le renforcement du pilotage frontal des séances par le manuel, numérisé et projeté : (e5) «Je scanne beaucoup, des livres entiers, le fichier de maths, tous les albums, les cahiers d'activité ».L'enjeu déclaré est d'assurer un meilleur contrôle de l'activité individuelle des élèves et de leur fournir, par souci d'équité et de plus grande lisibilité, une projection magistrale du même support que celui sur lequel ils accomplissent leur activité individuelle : (e4) «Quand j'affiche un exo au tableau, je décortique, une page de livre se retrouve sur 7 ou 8 pages de logiciel. Pour que ce soit lisible, je prends beaucoup de pages (logiciel IWS). »

D'autres démarches font apparaître un effort vers la construction systématique et originale de supports de séquence pédagogique, comme instrument de gestion de classe. Dans un cas de préparation de ressources pour une séance de conjugaison l'enseignante ne cherche pas la bonne solution technique de manipulation, mais celle «qui marche » en fonction de l'objectif visé, quitte à multiplier à outrance les manipulations : (e3) «Là aussi, ça doit exister, mais je sais pas comment faire, mais je veux faire un cadre pour faire une étiquette. Ce que je fais, c'est que je mets une forme tout autour. Dans ce type de situation, je n'ai pas la réponse. Je veux faire une étiquette, je ne connais pas la procédure. Je tâtonne, je cherche, je ne trouve pas la réponse...Comme je ne trouve pas, je m'embête moins et je repasse par Word et je capture des images, pour moi c'est un gain de temps. Après je vais faire comme tout à l'heure, je découpe chaque cellule pour avoir des étiquettes avec des bordures. Je suis certaine qu'il y a plus rapide. Il faut quelques compétences informatiques ».

Dans 4 cas sur 6, les enseignants ont développé un système de gestion de fichier et de normalisation permettant de répondre à trois enjeux : le premier est la rapidité d'accès à un contenu (une leçon, une page numérisée, un lien) ; le second est liéà la perfectibilité et le réaménagement de la ressource, que l'enseignant remobilisera et modifiera par la suite, ou non ; le troisième est liéà la rupture avec les anciens supports avec lesquels l'enseignant a construit son identité professionnelle. (e1) «Ce qu'il a fallu que je travaille en termes d'organisation, c'est la nomenclature de tout ce que je mets sur mon ordinateur. C'était déjà en filigrane, mais aujourd'hui j'ai une 
présentation de mes documents bien claire, de façon à les retrouver sans faute, sans erreur possible. J'ai une norme, j'ai normalisé l'édition de mes titres. Je m'y retrouve, que ce soit sur le blog, sur le logiciel du TNI. Je sais que tel document sera identique sur n'importe quelle plate-forme ».

Cependant aucun ne déclare mutualiser ses ressources. Certaines tentatives se limitent au local et les efforts consentis pour produire se heurtent à des considérations institutionnelles perçues comme des contraintes : (e1) «Après j'avoue que je ne suis pas très sûr en termes de droits d'auteur, je me souviens assez peu à qui j'ai fait les emprunts et j'ai du mal à remettre les auteurs alors je limite. Je pense qu'un jour je ferai l'effort [...] Savoir d'oùça vient et à qui ça va... Je trouve que ces notions de droit d'auteur à ce niveau-là sont nulles et non avenues donc je n'en tiens pas compte. Ça vous empêche de délivrer votre propre travail ».

Le paradigme de la continuité entre l'intervention simultanée et frontale de l'enseignant, instrumentant son activité avec le manuel et le tableau et l'activité individuelle des élèves se maintient, voire se renforce, dans l'ensemble des configurations. L'art du manuel qui est l'apanage du maître d'école trouve avec le TNI une nouvelle dimension, celle de la projection et de la manipulation directe. La fonction panoptique de contrôle du tableau se combine bien avec celle, de guidance pédagogique, induite par le manuel. Au maître de régler le dispositif technique pour que l'attention de l'élève soit soutenue.

L'appropriation du TNI par l'enseignant est donc empirique, relève du bricolage, et les cheminements parfois compliqués, mais tous tendus vers un résultat visible : «ce que je montre aux élèves ». L'intention de l'enseignant est d'aboutir à un objet finalisé, support d'une activité pédagogique plus ou moins complexe. La construction de cet objet est déterminée par une intention donnant lieu à des actions sur un système d'instruments dont le TNI fait partie (ordinateur personnel, ordinateur de classe, internet, appareils photos numériques, scanner, imprimantes, ressources papier). Les actions mises en œuvre se traduisent par des opérations plus ou moins guidées par des intentions et un processus de rétroactions et d'ajustements successifs.

\subsubsection{Enjeux axiologiques}

Quand il s'agit de discuter des valeurs de l'école, les positions sont plus marquées. Les enseignants font part du caractère inévitable d'une formation aux TICE et de leurs usages en classe, pour une école inscrite dans 


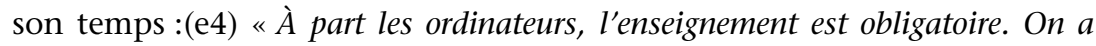
une salle informatique. J'ai toujours fait de l'informatique avec eux dans le cadre de l'enseignement obligatoire. ";(e6) «Oui, mais bon, moi je m'y suis mise... chaque fois que j'ai avancé dans le domaine de l'informatique, c'est par contraintes professionnelles, ce n'est pas par choix idéologique, ni de désir de me lancer là-dedans[...].Est-ce que c'est l'école qui apporte quelque chose par rapport à l'informatique aux élèves, ou est-ce que ce sont les élèves qui nous font bouger?[...]Voilà, donc quelque part j'ai l'impression que par moment, c'est nous qui nous adaptons, et puis après, j'en reviens à ce que je disais, et on utilise ce qu'on veut faire passer[...] Par moments quand on, surtout quand on commence à avoir des élèves un peu grands, euh... il y a un décalage entre nous à l'école».

Les enjeux liés à l'évolution des pratiques sociales et culturelles des élèves reviennent dans les témoignages. Pour les enseignants enquêtés, l'école doit être un lieu où l'on forme les jeunes à la société dans laquelle ils vivent, donc, en prenant en compte les technologies informatisées. Cet aspect transcende en partie les positions des enseignants plus en retrait, plus réservés vis-à-vis des TICE, mais impliqués sur les enjeux liés à l'éducation des jeunes.

\section{Discussion et perspectives}

Notre questionnement initial a porté sur le renforcement possible des formes magistrales d'enseignement $\mathrm{du}$ fait de l'existence des schèmes d'usage déjà rodés avec le tableau inerte, noir ou blanc. Il évoquait également une contradiction possible entre une volonté institutionnelle d'innover par les technologies et le possible renforcement de formes classique d'enseignement avec les technologies retenues. A ce stade, la recherche menée permet d'apporter une première réponse sur le processus à l'œuvre lors de l'adoption de ce dispositif technique, qui n'inscrit pas les enseignants dans une rupture, mais les amène toutefoisà produire un discours d'intelligibilité pour que ces pratiques deviennent stables et régulières. Cette réponse,éclairée par le modèle AFRI, amène également à poser quelques perspectives de recherche et des pistes d'accompagnementdes enseignants.

Les résultats obtenus sont transitoires et ne valent que pour les enquêtes réalisées et doivent être encore précisés. Trois types de facteurs contribuent à une entrée dans les usages du TNI: la conscience d'enjeux liés au développement du numérique dans les pratiques sociales et de l'éducation des élèves à ces nouveaux environnements d'activité ; 
l'acceptation d'un dispositif technique complexe et de la prise en compte des contraintes posées ;une prédisposition et de la curiosité.

Pour adapter le TNI à leurs pratiques de classe, les enseignants s'appuient sur ce qu'ils font déjà,le processus d'appropriation allant des considérations pédagogiques et didactiques vers les fonctionnalités qui en permettent la mise en œuvre. Les enseignants qui développent des pratiques nouvelles avec le TNI, sont inscrits dans un processus de genèse instrumentale qui les amène le plus souvent à reconsidérer certains aspects pédagogiques et didactiques de leur pratique de classe. Cette rétroaction entre la pratique et le dispositif technique est un des facteurs de pérennisation de l'usage du TNI. Autrement dit, l'accompagnement des enseignants dont la classe est équipée pourrait leur permettre de les aider à regarder au-delà des routines qui s'installent et qui sont fortement ancrées dans les usages habituels du tableau.

Le modèle AFRI nous permet de souligner les contraintes liées aux questions institutionnelles, matérielles et humaines auxquelles les enseignants interviewés sont soumis. Ils expriment un besoin de formation et d'échanges et interrogent le sens de leurs pratiques avec des instruments numériques. Le modèle pointe les tensions et contraintes qui font système et dans lequel l'enseignant peut en partie agir et qui l'oblige à prendre position : ses représentations personnelles sur les TICE et sur l'école; sa capacitéa formuler des demandes d'aides, à s'impliquer et se former. Le TNI amène ainsi l'enseignant à repenser un objet qui ne l'était plus : le tableau de classe.

Si nous revenons sur les enjeux institutionnels, le fait d'équiper les classes de TNI ne suffit pasà apporter aux enseignants les moyens d'une éducation «aux et par » les technologies informatisées pour reprendre le discours ministériel actuel à propos du numérique ${ }^{4}$. Cette condition est nécessaire, mais non suffisante. Certes, l'équipement matériel des classes est un marqueur d'un effort collectif et donne le sentiment d'une modernisationde l'école. Cependant, le TNI jette l'enseignant dans l'embarras : l'impression de ne pas s'en servir comme il faut, parfois, de faire moins bien qu'avec le tableau classique, avec un sentiment de frustration. Pour sortir de cette situation, certains enseignants mobilisent des moyens personnels d'autoformation, de réflexivité, de production de ressources pour faire le saut qualitatif et quantitatif nécessaire pour une appropriation assumée et dynamique de l'instrument. Sur ce dernier point, il apparaît que le niveau des compétences instrumentales et de connaissances infor- 
matiques chez plusieursenseignants contribue à une grande stabilité de leurs pratiques et à l'existence de démarches d'invention. Leur déficit chez d'autres enseignants fragilise les pratiques. Par ailleurs, la régularité de certaines utilisations du TNI ne permet pas de préjuger du type de ressources mobilisées ni de la qualité pédagogique des pratiques mises en œuvre.

Sur un autre plan, rappelons que les «techniques usuelles de l'information et de la communication » font partie du socle commun de connaissances et de compétences de l'école, sans pour autant être une discipline à enseigner. Autrement dit, leur acquisition par les élèves est fortement dépendante de la manière de travailler en classe. L'enseignant dans sa classe peut éprouver des difficultés à mettre en œuvre des compétences transversales pour lesquelles aucun espace disciplinaire n'est dégagé.

A partir de ces résultats, quelques directions pour un accompagnementdes enseignants qui se voient dotés de dispositifs complexes à mettre en œuvre pourraient être identifiés: le développementde compétences techniques relatives aux dispositifs mobilisés ; le développement d'espaces et de temps d'analyse de la pratique; le développement de stratégies de production de ressources dédiées à ces dispositifs techniques.

\section{BIBLIOGRAPHIE}

AKRICH M., CALLON M., LATOUR B. (1988). A quoi tient le succès des innovations? 1: L'art de l'intéressement ; 2 : Le choix des porte-parole. In Gérer et Comprendre. Annales des Mines, Gérer et comprendre. Consulté en mai 2013 à l'adresse http://halshs.archives-ouvertes.fr/halshs-00081741

ALAMI S., DESJEUX D., GARABUAU-MOUSSAOUI I. (2009). Les méthodes qualitatives - Paris : PUF

ARNOTT (2004). Computers to replace school blackboards. Consulté en mai 2013 à l'adresse http://www.computing.co.uk/ctg/news/1860992/computersreplace-school-blackboards

AVERIS D., GLOVER D., MILLER D. (2005). Motivation: the contribution of interactive whiteboards to teaching and learning in mathematics. Working group 9 Tools and Technology in Mathematical Didactics, 1051.

BARDIN, L. (1977). L'analyse de contenu. Paris : PUF.

BARON G.-L., BOULC'H L.,SEDOOKA A. (2011). Revue de question sur les technologies de l'information et de la communication à l'école primaire. Projet OPPIDUM. Ville de Saint-Maur-des-Fossès

BARON G.-L., BRUILLARD E. (1996). L'informatique et ses usagers dans l'éducation. Paris : PUF.

BOULC'H L., BARON G.-L. (2011). Connaissances et représentations du Tableau Numérique Interactif chez les futurs professeurs des écoles. Présentéà Didapro4 - 
Dida\&STIC, Patras (Grèce) Consulté en mai 2013 à l'adresse: http://www.ecedu.upatras.gr/didapro/final/actes/Boulc\%27hBaronDidapro2011.pdf

BECTA (2005). Review: Evidence on the progress of ICT in Education. Consulté en mai 2013 à l'adresse http://dera.ioe.ac.uk/1428/

BETRANCOUR, M. (2007). Pour un usage des TIC au service de l'apprentissage. CNDP, Hors série. Consulté en mai 2013 à l'adresse : http://tecfalabs.unige.ch/mitic/system/files/Betrancourt_UsagesTIC_apprentissage. pdf

BÉZIAT J. (2011). Se former aux TICE - Discours et représentations (p. 1096123). Présentéà Didapro4 - Dida\&STIC, Patras (Grèce) ; Consulté en mai 2013 à l'adresse: http://www.ecedu.upatras.gr/didapro/final/actes/BeziatDidapro2011.pdf

BÉZIAT J., VILLEMONTEIX F. (2012). Les technologies informatisées à l'école primaire. Déplacements et perspectives. In Colloque JOCAIR 2012. Présentéà Journées Communication et Apprentissage instrumentés en Réseau, 6-8 sept. Amiens. Consulté en ligne : http://edutice.archivesouvertes.fr/docs/00/77/98/95/PDF/BeziatVillemonteix.pdf

BILLOUET P. (2007). Tableau scolaire et modernité. Recherches en Education, $\mathrm{n}^{\circ} 17$.

BOULC'H L., BARON G.-L. (2011). Connaissances et représentations du Tableau Numérique Interactif chez les futurs professeurs des écoles. Actes du quatrième colloque international DIDAPRO 4 - Dida\&STIC, 24-26 octobre 2011, Université de Patras.

CHAPTAL A. (2010). "Paint it Black ». Eléments de réflexion sur les TICE en Angleterre à l'occasion du BETT 2010. Cap Digital.

DAGUET H., WALLET J. (2012). Du bon usage du « non-usage » des TICE. Recherches \&éducations, Vol. 6, p 35-53.

CUBAN L. (2001). Oversold and Underused: Computers in the Classroom. Cambridge, MA : Harvard University Press

DE LIÈVRE B., MOULIN B. (2008). L'analyse de l'usage des médias en classe comme vecteur de réflexion pédagogique. Consulté en mai 2013 à l'adresse http://hdl.handle.net/2013/UMONS-DI:oai:di.umons.ac.be:1195

DEPOVER C., (2009). La recherche en technologie éducative : fondements et approches, in Depover C., dir., La recherche en technologie éducative, un guide pour découvrir un domaine en émergence,édition des archives contemporaines, Agence Universitaire de la Francophonie, paris, 2009, 86p, chapitre 1, p5-13, ISBN : 978-28130-0008-8.

DEPOVER C., STREBELLE A. (1997). Un modèle et une stratégie d'intervention en matière d'introduction des TIC dans le processus éducatif. L'ordinateur à l'école: de l'introduction à l'intégration, p. 73-98.

DUROISIN N., TEMPERMAN G., DE LIÈVRE B. (2011). Effets de deux modalités d'usage du tableau blanc interactif sur la dynamique d apprentissage et la progression des apprenants. In Actes de la conférence EIAH 2011, p. 257-269. Consulté en mai 2013 à l'adresse http://telearn.archives-ouvertes.fr/hal-00609090/

DWYER D. C., RINGSTAFF C., HAYMORE J., SANDHOLZ P. D. (1994). Apple classrooms of tomorrow. Educational Leadership, Vol. 51 n $^{\circ} 7$, p. 4-10.

FLUCKIGER C., BART D. (2012). L'introduction du B2i à l'école primaire: évaluer des compétences hors d'une discipline d'enseignement? Questions Vives. Recherches en éducation, Vol. $7 \mathrm{n}^{\circ}$ 17. Consulté en novembre 2013 à l'adresse $\mathrm{http}: / / q u e s t i o n s v i v e s . r e v u e s . o r g / 1006$ 
HIGGINS S., FALZON C., HALL I., MOSELEY D., SMITH F., SMITH H., WALL K. (2005). Embedding ICT in the literacy and numeracy strategies: final report.

IGEN. (2011). École numérique rurale - L’opération École numérique rurale Éduscol. Consulté enmars 2012, à l'adresse http://eduscol.education.fr/cid56257/ecole-numerique-rurale.html

INGOLD, T. (2010). L'outil, l'esprit et la machine : Une excursion dans la philosophie de la "technologie". Techniques \& Culture, Vol. 2 n54-55, p. 291-311.

JEUNIER B., CAMPS J.-F., GALY-MARIÉ E., TRICOT A. (2005). Expertise relative aux usages du tableau blanc interactif en école primaire. DT/SDTICE. Consulté en mai 2013 à l'adresse http://www.tableauxinteractifs.fr/wpcontent/uploads/docs/expertise-tbi-2005.pdf

MAGNAT E. (2012). Visualization and Manipulation of English Sounds on an Interactive Whiteboard at Primary School. in Actes du colloque EARLI SIG 2 (De Vries, Scheiter, eds), 28-31 août 2012, p.130. Université de Grenoble

MILES M. B., HUBERMAN, A. M. (2003). Analyse des données qualitatives. De Boeck Supérieur.

MORINEAU, T. (2001). Eléments pour une modélisation du concept d'affordance. In Actes du Colloque EPIQUEp. 83-95).

RABARDEL P. (1995). Les hommes et les technologies.Paris: Armand Colin.

ROYAL SOCIETY. (2013). Shut Down or restart.Consulté en mai 2013 à l'adresse http://royalsociety.org/education/policy/computing-in-schools/report/

SMITH H., HIGGINS S., WALL K., MILLER J. (2005). Interactive whiteboards: boon or bandwagon? A critical review of the literature.Journal of computer assisted learning,Vol. $21 \mathrm{n}^{\circ} 2$, p. 91-101.

SOMEKH B., HALDANE M., JONES K., LEWIN C., STEADMAN S., SCRIMSHAW P., ... others. (2007). Evaluation of the primary schools whiteboard expansion project. London: Report to the Department for Education and Skills.

THOMPSON J., FLECKNOE M. (2003). Raising attainment with an interactive whiteboard in Key Stage 2. Management in Education, Vol. $17 \mathrm{n}^{\circ} 3$.

VILLEMONTEIX F. (2011). Informatique scolaire à l'école primaire. Spécificités et devenir du groupe professionnel des animateurs TICE. Paris: L'Harmattan.

VILLEMONTEIX F., STOLVIJK C. (2011). Processus d'adoption du TNI: quelle part de soi? In Didapro 4 - Dida\&STIC. Sciences et technologies de l'information et de la communication (STIC) en milieu éducatif. Université de Patras (Grèce).

\footnotetext{
1 L'enquêteETIC2010 révèle un taux d'équipement d'un ordinateur pour 9 élèves en moyenne et de 1 TNI pour 500 élèves.

${ }^{2}$ Voirdonnées-

del'enquêteSTEPS(Etudedel'impactdestechnologiesdanslesécolesprimaires).CommissionEuropée nne(2007).

3 Site OPPIDUM, consulté en avril 2013: http://oppidum.crdpcreteil.fr/spip.php?rubrique264

${ }^{4}$ Voir note aux recteurs du ministre Peillon, « déclinaison au niveau académique de la stratégie numérique du ministère ", datée de juin 2013 disponible ici : http://www.cafepedagogique.net/lexpresso/Documents/docsjoints/mencircuinfo.pdf
} 